\title{
Analysis and Research of a Method of Improving the Accuracy of the Fundamental Matrix
}

\author{
Yongxiang zhang ${ }^{1,2}$, Tieying $\mathrm{Mu}^{1,}$, , Weigong Zhang ${ }^{1,2}$, Peiqiang $\mathrm{Gu}^{1}$ \\ ${ }^{1}$ College of Information Engineering, Capital Normal University, Beijing 100048, China \\ ${ }^{2}$ Beijing Engineering Research Center of High Reliable Embedded System, Beijing 100048, China \\ a1403077723@qq.com
}

Keywords: fundamental matrix, computer vision, SURF, wrong matching, image noise, RANSAC.

\begin{abstract}
The fundamental matrix contains all of a camera's inner and outer parameters. So estimating the fundamental matrix is an important issue in 3D reconstruction and even the whole field of computer vision. As for the accuracy, stability and universality in calculating the fundamental matrix, this paper gets the coordinates of matched points using SURF algorithm at first, then compares and analyses the results of the two factors affecting the estimation results, such as wrong matching and image noise. Experimental results show that using RANSAC algorithm to remove the wrong matching and the less-noise images can achieve higher accuracy of the fundamental matrix.
\end{abstract}

\section{Introduction}

Obtained from two different viewpoints between two images of the same scene, there exist important relations of geometric constraint, namely epipolar geometry [1]. The epipolar geometry can be described by a $3 \times 3$ singular matrix called fundamental matrix. Fundamental matrix estimation is the foundation of 3D reconstruction, motion estimation, camera calibration, and matching and tracking [1][2]. So, the fundamental matrix computation plays an important role in the whole computer vision.

The fundamental matrix expresses the corresponding relationship of matched points between images from mathematical perspective. First of all, we need to extract the feature points of images and match them [3]. In this paper, a lot of corresponding points are gained after extracting and matching feature points by means of the SURF algorithm. When there are more than eight pairs of matched points, we calculate the fundamental matrix through the improved eight-point algorithm [4], with the residual error of the fundamental matrix as a measure of its precision.

There are many factors affecting the results of fundamental matrix estimation. This article will focus on the two factors such as matching errors of the feature points and noise interference to the selected images. Through the final contrast of average residual error of the fundamental matrix, it can be found that for the images of less noise, when adding RANSAC algorithm in SURF algorithm, the accuracy of matched points will be greatly improved and thus we can get the fundamental matrix of higher precision.

\section{Extracting and Matching of Feature Points}

In this paper, we use the algorithm of SURF as feature extraction algorithm. The main process is divided into the extraction of feature points and generation of SURF feature descriptor [5]. SURF algorithm is an improvement based on SIFT algorithm, with better performance and faster execution [5].

\section{Feature Extracting.}

The detection of SURF operator is based on the scale of the "inverted pyramid" structure space [6], and obtains feature points through the Hessian matrix [7].

The Hessian matrix of a point of the image $\mathrm{I}(\mathrm{x}, \mathrm{y})$ of $\sigma$ scale is defined as: 


$$
H=\left[\begin{array}{ll}
L_{x x}(x, \sigma) & L_{x y}(x, \sigma) \\
L_{x y}(x, \sigma) & L_{y y}(x, \sigma)
\end{array}\right]
$$

$\mathrm{L}_{\mathrm{xx}}(\mathrm{x}, \sigma), \mathrm{L}_{\mathrm{xy}}(\mathrm{x}, \sigma), \mathrm{L}_{\mathrm{yy}}(\mathrm{x}, \sigma)$ is the second-order derivative of gaussian function and the two-dimensional convolution.

SURF algorithm simplifies the second-order differential template through box filter, and calcutes the convolution of the image and box filter of different scales through integral image in order to get the determinant of the simplified Hessian matrix:

$$
\operatorname{Det}(\mathrm{H})=\mathrm{D}_{\mathrm{xx}} \mathrm{D}_{\mathrm{yy}}-\left(0.9 \mathrm{D}_{\mathrm{xy}}\right)^{2}
$$

$D_{x x}, D_{y y}, D_{x y}$ are the approximations of $L_{x x}, L_{y y}, L_{x y}$ respectively.

We can calculate the determinant $\operatorname{Det}(\mathrm{H})$ of each pixel using the formula above, then use non-maximum suppression in the three-dimensional neighborhood of each extreme value point, finally choose the candidate feature points.

\section{Feature Description.}

In order to describe the descriptor with rotation invariance, we determinate the feature points through the extremum of Harr Wavelet response in round neighborhood [5][6], then choose 20s $\times 20 \mathrm{~s}$ square area centered on feature points, dividing this window into $4 \times 4$ sub-domain, and calculate the sum of Harr Wavelet response and sum of absolute value of $\mathrm{x}, \mathrm{y}$ direction in every sub-domain, There will be the 64-dimensional feature vector, finally get the feature descriptor via normalization.

\section{Feature Matching.}

Under normal circumstances, there exists much mismatching using space distance as matching criteion only. We combine the gray similarity of feature points and their neighborhood pixels with the space distance contraints as a rule of corresponding points, which can effectively improve the accracy of matching.

\section{Fundamental Matrix Estimation}

\section{Fundamental Matrix.}

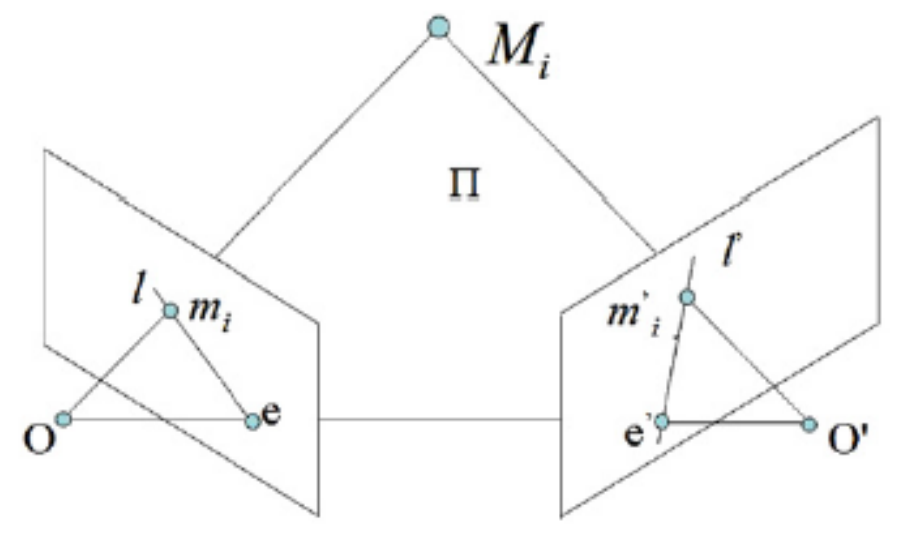

Fig.1 Epipolar geometry

As illustrated in Fig.1, assume that two pictures of the same scene are $\mathrm{I}, \mathrm{I}^{\prime}, \mathrm{m}_{\mathrm{i}}=\left(\mathrm{x}_{1}, \mathrm{y}_{1}, 1\right)^{\mathrm{T}}$ and $\mathrm{m}_{\mathrm{i}}{ }^{\prime}=\left(\mathrm{x}_{2}, \mathrm{y}_{2}, 1\right)^{\mathrm{T}}$ are respectively projective points of space point $\mathrm{M}_{\mathrm{i}}$ in two pictures. For an arbitrary point $\mathrm{m}_{\mathrm{i}}$ in the first image, its correspondence in the second image $\mathrm{m}_{\mathrm{i}}{ }^{\prime}$, must lie on the epipolar line $\mathrm{l}^{\prime}\left(\mathrm{l}^{\prime}=\mathrm{Fm}_{\mathrm{i}}\right)$ in the second image. The epipolar geometry relation is satisfied as follows, where $\mathrm{F}$ is the fundamental matrix.

$$
\mathrm{m}_{\mathrm{i}}^{\prime \mathrm{T}} \mathrm{Fm}_{\mathrm{i}}=0, \quad \mathrm{~F}=\left[\begin{array}{lll}
\mathrm{f}_{11} & \mathrm{f}_{12} & \mathrm{f}_{13} \\
\mathrm{f}_{21} & \mathrm{f}_{22} & \mathrm{f}_{23} \\
\mathrm{f}_{31} & \mathrm{f}_{32} & \mathrm{f}_{33}
\end{array}\right]
$$

We rewrite $\mathrm{F}$ in the following way 
Af $=0$, where $A=\left(x_{2} x_{1}, x_{2} y_{1}, x_{2}, y_{2} x_{1}, y_{2} y_{1}, y_{2}, x_{1}, y_{1}, 1\right), f=\left(f_{1}, f_{2}, f_{3}, f_{4}, f_{5}, f_{6}, f_{7}, f_{8}, f_{9}\right)$

When there are multiple sets of points, it can establish some equations, and calculate fundamental matrix through solving equations [8].

\section{Improved Eight-point Algorithm.}

When the number of corresponding points is greater than or equal to eight, the fundamental matrix can be solved by least square method. Eight-point algorithm converts this problem to the following questions:

$\min \|\operatorname{Af}\|$, where constraint condition is $\|\mathrm{f}\|=1$

First, we make singular value decomposition to the coefficient matrix A in the formula (4)[10] $\mathrm{A}=\mathrm{UDV}^{\mathrm{T}}$, the last column of vector $\mathrm{V}$ is the fundamental matrix $\mathrm{F}$. Then make rank-two constraint to it, namely, making singular value decomposition to $\mathrm{F}=\operatorname{Udiag}\left(\mathrm{s}_{1}, \mathrm{~s}_{2}, \mathrm{~s}_{3}\right) \mathrm{V}^{\mathrm{T}}$.

In order to improve the accuracy of fundamental matrix estimation, Hartley made normalized transformation to image coordinates based on the classical eight-point algorithm. After normalization, the precision of fundamental matrix calculation has greatly improved.

\section{Factor Analysis Influencing the of Fundamental Matrix}

In the process of fundamental matrix estimation, there are so many factors which can influence the precision of final results, such as the degree of images affected by noise, and mismatching phenomena caused by matching algorithm. They will have a great effect on the accuracy of corresponding points, and then influence the precision of following eight-point algorithm; In addition, Eight-point algorithm is just a kind of estimation algorithm, so normalized transformation to image coordinates, the selecting principle of eight set of points will also affect the least square solution of equation. This section will conduct effect comparison and analysis to the two factors of affecting matched points precision such as mismatching and noise interference.

\section{Mismatching.}

The matching method mentioned in 1.3 inevitably appears the mismatching phenomenon, and it has a great influence on the next work. The input of eight-point algorithm is eight couple of points, so once mismatching appears, it will directly influence the result of fundamental matrix. After completing the initial image matching, it need also remove the wrong matched points for further. This article chooses the most commonly used RANSAC algorithm[11] to remove false matching, which can improve the accuracy of the matching, thus improving the accuracy of the fundamental matrix.

\section{Noise Interference.}

The degree of different images affected by noise will be different. Even the same image, affected by noise, information and parameters of the image itself will also change, which will affect the feature points extraction and matching results. For the image of less noise, the influence on feature point extraction is smaller, the matching success rate higher, and the accuracy of the fundamental matrix better.

\section{Results and Analysis of Experiments}

According to the above method to estimate fundamental matrix, first we need to get two images taken from different perspective, then extract and describe the image feature, and complete the match via SURF algorithm. Finally we use eight-point algorithm to estimate the fundamental matrix F. We calculate the average residual error [12] as a measure of precision. The higher the fundamental matrix precision, the less the average residual error value. As is shown in formula (6), $\mathrm{N}$ is the number of matching points, $\mathrm{I}_{\mathrm{i}}, \mathrm{I}_{\mathrm{i}}$ are homogeneous coordinates of the matching points of two images respectively.

$$
\mathrm{r}=\frac{1}{\mathrm{~N}} \sum_{\mathrm{i}=1}^{\mathrm{N}}\left|\mathrm{I}_{\mathrm{i}}^{\mathrm{T}} \mathrm{FI}_{\mathrm{i}}\right|
$$


Here $\mathrm{N}=8$, for the eight sets of point correspondences chosen by the algorithm, we respectively calculate the residual error, taking an average to get average residual error, finally mapping the relationship curve with the number of points.
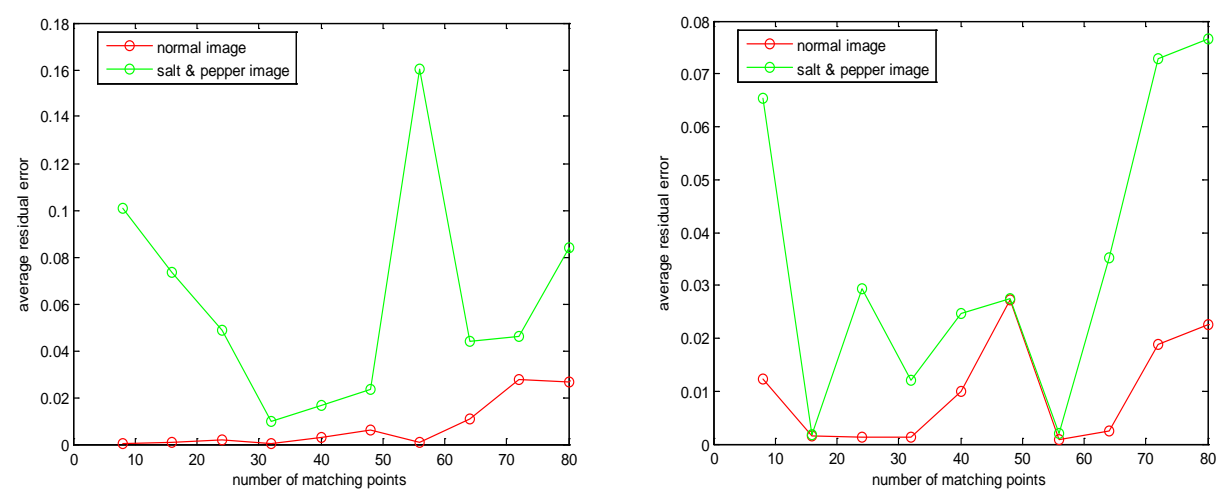

Fig.2 Average residual error curve of images of different quality

Table 1 The average contrast of average residual error

\begin{tabular}{ccccc}
\hline & 1 & 2 & 3 & 4 \\
\hline normal images & 0.0097 & 0.0080 & 0.0118 & 0.0068 \\
salt \& pepper images & 0.0332 & 0.0496 & 0.0303 & 0.0297 \\
\hline
\end{tabular}

As is shown in Fig.2, there is the curve contrast of average residual error of images of different quality after a few times performances. It can be seen that influenced by the number of the selected matched points, the average residual error value has a big ups and downs, and contrasts not obviously in a certain number. Overall the curve of the average residual error of the normal images is in the curve of the salt and pepper images below.

In order to show the problem more quantitatively, we take out the maximum and minimum of all the average residual error value, and take the average, as is shown in Table 1 . These contrasts show that the value of average residual error is smaller in images of less noise, namely higher accuracy of the fundamental matrix estimation.
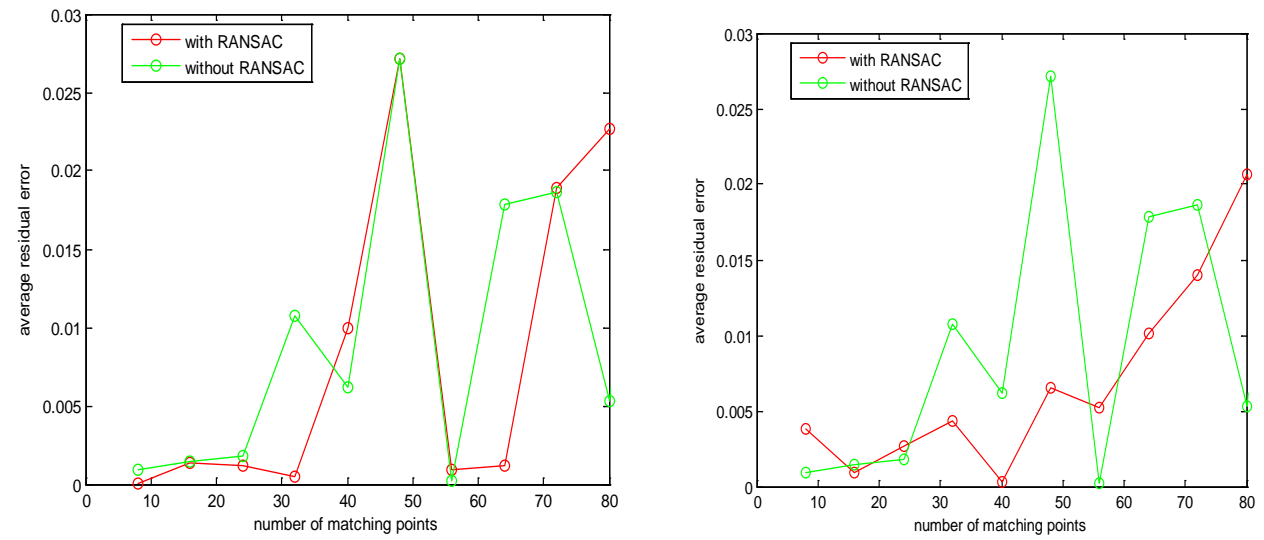

Fig.3 average residual error curve of images with and without RANSAC

Table 2 The average contrast of average residual error

\begin{tabular}{ccccc}
\hline & 1 & 2 & 3 & 4 \\
\hline with RANSAC & 0.0079 & 0.0067 & 0.0062 & 0.0077 \\
without RANSAC & 0.0148 & 0.0148 & 0.0148 & 0.0148 \\
\hline
\end{tabular}

As is shown in Fig.3 and Table 2, there is the curve contrast of average residual error of images with and without RANSAC algorithm after a few times performances. For the case of no RANSAC 
algorithm, the same images have the same feature points, and it will not change every time, so are values of the fundamental matrix and average residual error. For the case with RANSAC algorithm, there will be randomness in the process of getting rid of the mismatching. So it cannot guarantee the exactly same matched points after removing the false matching, so are values of the fundamental matrix after every execution and average residual error.

From the general trend of the curve and quantitative analysis, it can be indicated that the average residual error of images will be smaller with RANSAC algorithm, namely higher accuracy of fundamental matrix estimation. However, because of large fluctuation of values, it is inevitable appearing the opposite conclusion in individual points. So we can know that there is more or less error in estimation of the fundamental matrix.

\section{Conclusions}

This paper analyses the influence of two factors in estimating fundamental matrix, which are with RANSAC algorithm or without, noise of images. Results of a lot of simulation experiments indicate that for the images of less noise, when adding RANSAC algorithm in SURF algorithm, the accuracy of matched points will be greatly improved and thus we can get the fundamental matrix of higher precision.

\section{Acknowledgements}

The research work was supported by the National Science Foundation under grant 61472260 and 61402302, the Beijing Natural Science Foundation under grant 4143060, The Project of Construction of Innovative Teams and Teacher Career Development for Universities and Colleges under Beijing Municipality under grant No.IDHT20150507.

\section{References}

[1] Stackelberg H. The Theory of the Market Economy. Oxford: Oxford University Press, 1952.

[2] Miller, T., Friesz,T. \& Robin,R., Heuristic algorithms for delivered pric spatially competitive network facility location problem. Ann. Oper. Res., 34, pp. 177-202, 1992.

[3] Pieume, C.O, Fotso L.P. \& P. Siarry. A method for solving bilevel linear progremming problem. Journal of Information and Optimization Science, 29(2), pp. 335-358, 2008.

[4] Aryanezhad,M.B. \& Roghanian, E.A., Bilevel linear multi-objective decision making model with interval coefficents for suppy chain coordination. Iust Internation Journal of Engineering Science, 19(1-2), pp.67-74, 2008,

[5] Cheng-Min Feng \& Chieh-chao Wen, A fuzzy bi-level and multi-objective model to control traffic flow into the disaster area post-earthquake. Journal of the Eastern Asia Society for Transportation Studies, 6, pp. 4253-4268, 2005.

[6] Li He-Cheng, Wang Yu-Ping, An interpolation based genetic algorithm for sloving nonlinear bilevel programming problems.Chinese Journal of Computers, 31(6), pp.910-918, June 2008.

[7] Kalyanmoy Deb, Amrit Pratap, Sameer Agarwal \& Meyarivan, T.A., Fast and elitist multi-objective genetic algorithm: NSGA-II, IEEE Trans. Evol. Comp., 6(2), pp.182 - 197, 2002.

[8] Wang Yu-Ping, the Theory and Method of Evolutionary Computation, Beijing: Science Press, 2011.

[9] Farahi, M. H. \& Ansari, E. A new approach to solve muti-objective linear bilevel programming problems. Journal of Mathematics and Computer Science, 1(4), pp.313-320, 2010.

[10] Eman, O.E., Interactive bi-level multi-objective integer non-linear programming problem. Applied Mathematical Sciences, 5(65), 3221-3232, 2011. 